\title{
ENVIRONMENT DEPENDENCE OF DISK MORPHOLOGY OF SPIRAL GALAXIES
}

\author{
Hong Bae AnN \\ Department of Earth Science Education, Pusan National University, Pusan 609-735, Korea \\ E-mail : hbann@pusan.ac.kr \\ (Received October 27, 2013; Revised November 26, 2013; Accepted December 4, 2013)
}

\begin{abstract}
We analyze the dependence of disk morphology (arm class, Hubble type, bar type) of nearby spiral galaxies on the galaxy environment by using local background density $\left(\Sigma_{n}\right)$, projected distance $\left(r_{p}\right)$, and tidal index $(T I)$ as measures of the environment. There is a strong dependence of arm class and Hubble type on the galaxy environment, while the bar type exhibits a weak dependence with a high frequency of SB galaxies in high density regions. Grand design fractions and early-type fractions increase with increasing $\Sigma_{n}, 1 / r_{p}$, and $T I$, while fractions of flocculent spirals and late-type spirals decrease. Multiple-arm and intermediate-type spirals exhibit nearly constant fractions with weak trends similar to grand design and early-type spirals. While bar types show only a marginal dependence on $\Sigma_{n}$, they show a fairly clear dependence on $r_{p}$ with a high frequency of SB galaxies at small $r_{p}$. The arm class also exhibits a stronger correlation with $r_{p}$ than $\Sigma_{n}$ and $T I$, whereas the Hubble type exhibits similar correlations with $\Sigma_{n}$ and $r_{p}$. This suggests that the arm class is mostly affected by the nearest neighbor while the Hubble type is affected by the local densities contributed by neighboring galaxies as well as the nearest neighbor.
\end{abstract}

Key words : galaxies: structure — galaxies: formation — galaxies: morphology — galaxies: general

\section{INTRODUCTION}

The structure of a spiral galaxy is characterized by two primary components, disk and bulge of which radial luminosity distributions are well represented by an exponential profile (Freeman 1970) and $r^{1 / 4}$ law profile (de Vaucouleurs 1953), respectively. The ratio of the luminosities in these two components, the disk-to-bulge ratio, is a well defined quantity which can be used as a proxy of Hubble type. The spiral arms and bars are the secondary components displaying perturbations imposed on the disk. However, they are as important as the primary components, disk and bulge, because their structures are closely related to the internal and external properties of the galaxies (Elmegreen et al. 2011) and they are thought to drive secular evolution of the spiral galaxies (Kormendy 1979; Pfenniger \& Norman 1990; Friedli \& Benz 1993, 1995; Zhang 1996, 1998, 1999; Kormendy \& Kennicutt 2004).

In Hubble's morphological classification of galaxies, spiral galaxies are divided into subtypes, known as Hubble stages (early-, intermediate- and late-type), according to the dominance of bulge luminosity, arm openness, and patchness of the spiral arms. There are quantitative measures of the first and second properties, namely, bulge-to-disk ratio and the pitch angle of the spiral arms. They are considered to reflect the physical properties of the disk morphology. But it is difficult to directly relate the patchness with the physical properties of galaxies because it is affected by the distance of a galaxy. Thus, recent morphological studies of spiral galaxies consider the bulge-to-disk ratio and arm openness as the working criteria to distinguish the Hubble stage of spiral galaxies. Early-type spirals have tightly wound spiral arms and a large bulgeto-disk ratio, while the late-type spirals have loosely wound spiral arms and a small bulge-to-disk ratio.

However, the openness of the spiral arms is not the only property of spiral arm structures. Elmegreen \& Elmegreen $(1982,1987)$ introduced arm classes, which are sometimes denoted as AC, to distinguish the arm structures on the basis of the degree of symmetry and continuity together with the number of arms. The 12 arm classes introduced by Elmegreen \& Elmegreen (1982) were reduced to 10 arm classes, as two classes that are related to the presence of a bar (AC10) and companion (AC11) were removed. However, most previous studies divided the arm classes into two or three broad classes: (1) grand design $(\mathrm{G})$ and flocculent $(\mathrm{F})$ or (2) grand design (G), multiple-arm (M), and flocculent $(\mathrm{F})$. For the case of two broad classes, multiple-arm is considered as a subclass of the grand design arms. Grand design arms have two long symmetric arms, while flocculent arms have a large number of chaotic fragmented arms. Multiple arms exhibit intermediate properties between the grand design and flocculent arms. Some multiple-arm galaxies have short grand design arms in the inner parts and multiple arms in the outer parts. Some of the outer arms in multiple-arm galaxies are likely to be connected to produce pseudo- 

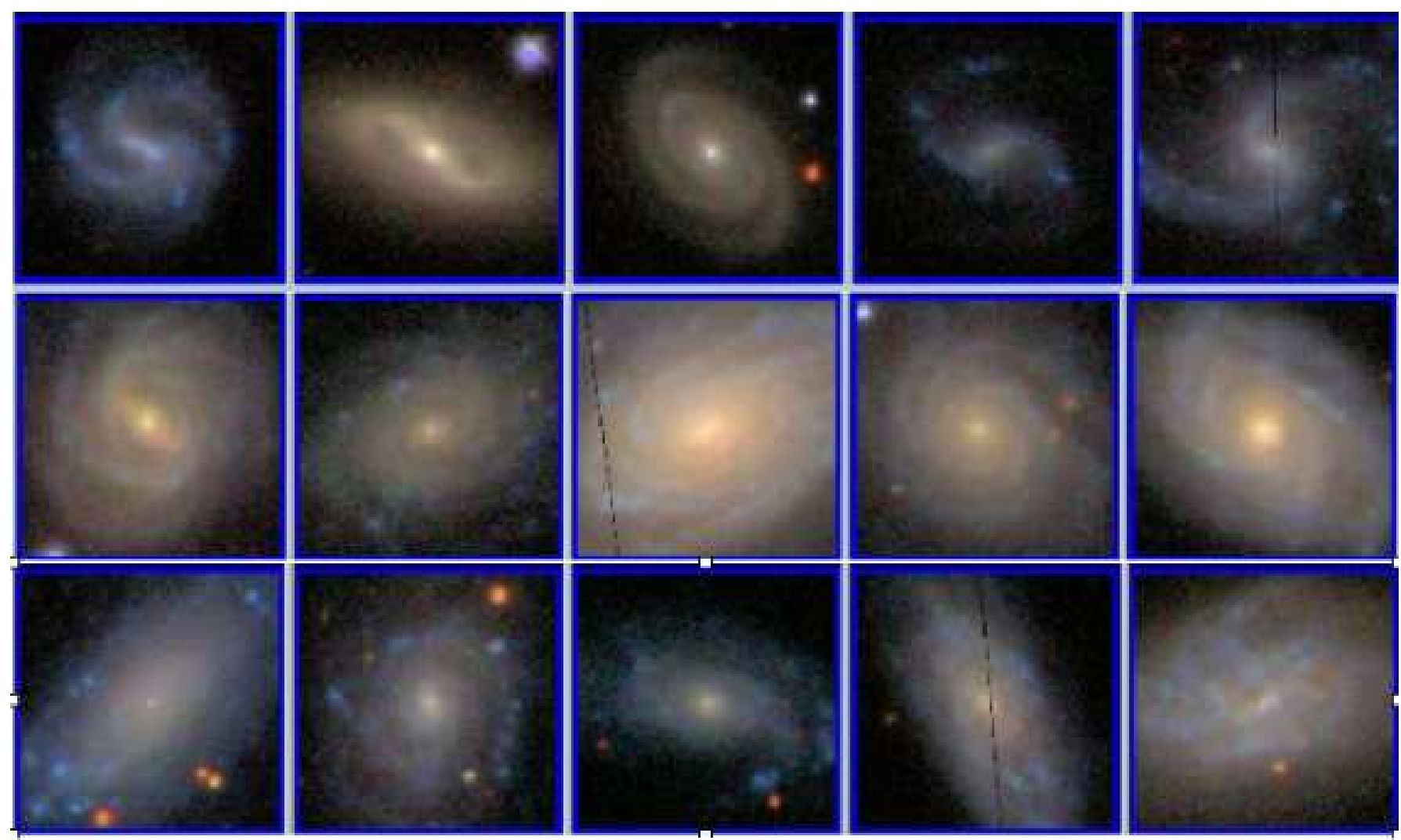

Fig. 1. - Sample of spiral galaxies. Grand design galaxies, multiple-arm galaxies and flocculent galaxies are displayed in the top panels, middle panels and bottom panels, respectively.

rings (Elmegreen \& Elmegreen 1982).

There have been many investigations regarding the relationships between arm classes and internal properties, including luminosity, color, disk-to-bulge ratio, and morpholgy. The variation in the mean luminosity and colors of galaxies across different arm classes is small (Ann \& Lee 2013). Conversely, considerable differences are observed in the galaxy size (Elmegreen \& Elmegreen 1987; Ann \& Lee 2013) and shape of rotation curves (Elmegreen 1990; Biviano et al. 1991). There seems to be little or no dependence of the arm classes on the star formation rate (Elmegreen \& Elmegreen 1986) and star formation related quantities such as the neutral hydrogen content (Romanishin 1985), CO surface brightness (Stark, Elmegreen, \& Chance 1987), and supernova rate (McCall \& Schmist 1986).

Arm classes exhibit some dependencies on the Hubble type (Elmegreen \& Elmegreen 1982; Choi \& Ann 2011; Ann \& Lee 2013). Flocculent galaxies are more likely to occur in late-type galaxies, while multiplearm galaxies are more frequent in early-type galaxies. Grand design galaxies are also more frequent in earlytype galaxies, but the correlation is much weaker than multiple-arm galaxies. There is a correlation between the arm class and bar type (SA, SAB, and SB). The fraction of grand design galaxies increases from $\mathrm{SA}$ to $\mathrm{SAB}$ to SB, while flocculent galaxies show the opposite correlation. Multiple-arm galaxies exhibit nearly constant fractions regardless of the bar type. If we consider the Hubble type and bar type together, early-type SB galaxies are likely to have a high fraction of grand design galaxies; however, late-type SB galaxies exhibit no such tendency (Elmegreen \& Elmegreen 1989).

The environment dependence of galaxy morphology is well understood for field galaxies (Goto et al. 2003; Park et al. 2007) as well as cluster galaxies (Dressler 1980). It is represented by the morphology-density relation (MDR) or morphology-radius relation (MRR). In other words, early-type galaxies (E/S0) are likely to be found in the dense environment or central regions of clusters, while late-type galaxies ( $\mathrm{Sp} / \mathrm{Irr}$ ) are more frequently observed in the under-dense regions or outer parts of clusters. The environment dependencies of the disk morphology of spiral galaxies, which are represented by arm class, Hubble type, and bar type, is of interest because the arm class is closely related to the driving mechanism of the spiral structures (Elmegreen 
\& Elmegreen 1986; Elmegreen et al. 2011). Grand design arms are thought to be formed by density waves (Lin \& Shu 1964; Bertin et al. 1989a,b), whereas flocculent arms are thought to be formed by stochastic self-propagated star formation (Gerola \& Seiden 1978; Seiden \& Gerola 1982). Thus, understanding the environment of galaxies with different arm classes provides some information about the conditions for various modes of star formation.

Early investigations into the environment dependence of arm classes (Elmegreen \& Elmegreen 1982; Elmegreen, Elmegreen, \& Dressler 1982) found that grand design galaxies are more frequent in high density regions, while flocculent galaxies favor low density regions. However, Giuricin, Mardirossian, \& Mezzetti (1989) reported that flocculent galaxies are more frequent in interacting or binary systems, which are likely to be present in dense environments. These contradictory claims may be due to poor statistics (Giuricin et al. 1994). A recent study of the arm class and local background density of nearby spiral galaxies (Ann \& Lee 2013) confirmed the earlier findings of Elmegreen \& Elmegreen (1982), Elmegreen, Elmegreen, \& Dressler (1982) and Elmegreen \& Elmegreen (1987). Ann \& Lee (2013) found that there is a weak trend of increase in the fraction of grand design galaxies with increasing local background density, while a stronger opposite trend of a decreasing fraction with increasing density was found for flocculent galaxies. They derived the local background density from the projected distances to the 5th nearest neighbor. However, as described by Muldrew et al. (2012), the local background densities derived using the $n$th nearest neighbor method depend on two parameters $\Delta V^{*}$ and $M^{*}$ which set the upper limit of the line of sight velocity difference between the target galaxy and its neighbor and the lower limit of the luminosity of the neighbor galaxies to be searched. Thus, it is necessary to choose a set of parameters $(n$, $\left.\Delta V^{*}, M^{*}\right)$ suitable for the local background density and relevant to the formation of spiral structures.

The natural choice for $M^{*}$ appears to be the limiting magnitude that defines the sample galaxies as a volume-limited sample. However, other values of $M^{*}$ are also possible if the selected $M^{*}$ is relevant to a physical process being investigated. In case of $\Delta V^{*}$, it would be different for galaxies in different environments because peculiar velocities depend on the large scale distribution of galaxies as well as nearby galaxies. Moreover, Park \& Choi (2009) argued that $\Delta V^{*}$ depends on the morphology of galaxies. They adopted

Table 1.

Frequency of arm class, Hubble type and bar type of nearby spiral galaxies.

\begin{tabular}{cccccccccc}
\hline & \multicolumn{3}{c}{ arm class } & \multicolumn{3}{c}{ Hubble stage } & \multicolumn{3}{c}{ bar type } \\
\hline & $\mathrm{G}$ & $\mathrm{M}$ & $\mathrm{F}$ & $\mathrm{e}$ & $\mathrm{i}$ & $\mathrm{l}$ & $\mathrm{SA}$ & $\mathrm{SAB}$ & $\mathrm{SB}$ \\
\hline $\mathrm{N}$ & 347 & 656 & 909 & 382 & 893 & 637 & 639 & 536 & 630 \\
\hline
\end{tabular}

$\Delta V^{*}=400$ and $600 \mathrm{~km} / \mathrm{s}$ for late- and early-type galaxies, respectively, to search for the nearest neighbor galaxies on the basis of the pairwise velocity difference between the target galaxy and their neighbors (Park, Gott, \& Choi 2008; Park \& Choi 2009). Since we are dealing with galaxies in a variety of environments, a proper value of $\Delta V^{*}$ is yet to be determined. In addition to this, it is quite likely that disk morphology correlates well with other environment measures than local background density.

The purpose of the present study is to determine which measures of environment are closely related to the disk morphology. To do this, we first explore the dependence of local background densities on the neighbor search parameters, $M^{*}, \Delta V^{*}$, and $n$, using the Sloan Digital Sky Survey (SDSS) Data Release 7 (DR7). Subsequently, we study local background densities of spiral galaxies, for which arm class, Hubble type, and bar type are known. We look for the appropriate set of $n, M^{*}$, and $\Delta V^{*}$ that we could use to derive the local background densities relevant to the disk morphology.

In Section 2, we describe the observational data and method of arm classification. Local background density dependence of disk morphology with effects of neighbor search parameters on the local background densities are presented in Section 3. We descrive other measures of local environment in Section 4. Discussion on the relation between the disk morphology and the local environment is given in Section 5 and conclusions of the present study are given in Section 6 followed by acknowledgements in the last section.

\section{OBSERVATIONAL DATA}

The present sample of local galaxies was extracted from the Korea Institute for Advanced Study Value Added Galaxy Catalog (KIAS VAGC), which provides the basic data of galaxies such as coordinates, redshifts, magnitudes, and colors along with the broad morphological types determined by automated classifiers (Park \& Choi 2005). See Choi, Han, \& Kim (2010) for a detailed description of the KIAS VAGC. Since the KIAS VAGC is based on the SDSS DR7, the present sample of galaxies is a flux-limited sample with a limiting magnitude of $r=17.7$. Thus, the galaxies with $z<0.02$ in the present sample (13762 galaxies) is complete up to $M_{r} \approx-16.1$. The sample of spiral galaxies used for the analysis of the environment dependence of disk morphology is a subsample of the present sample, for which arm classes, Hubble types, and bar types are given in (Ann \& Lee 2013). The numbers of arm class, Hubble type and bar type are listed in Table 1. Bar types of 107 spiral galaixes were not determined due to high inclinations. We present the sample images of arm classes in Fig. 1. We used the parent sample of 13762 galaxies as the basic data set for the environment analysis.

Fig. 2 shows the $u-r$ versus $M_{r}$ diagram of 13762 


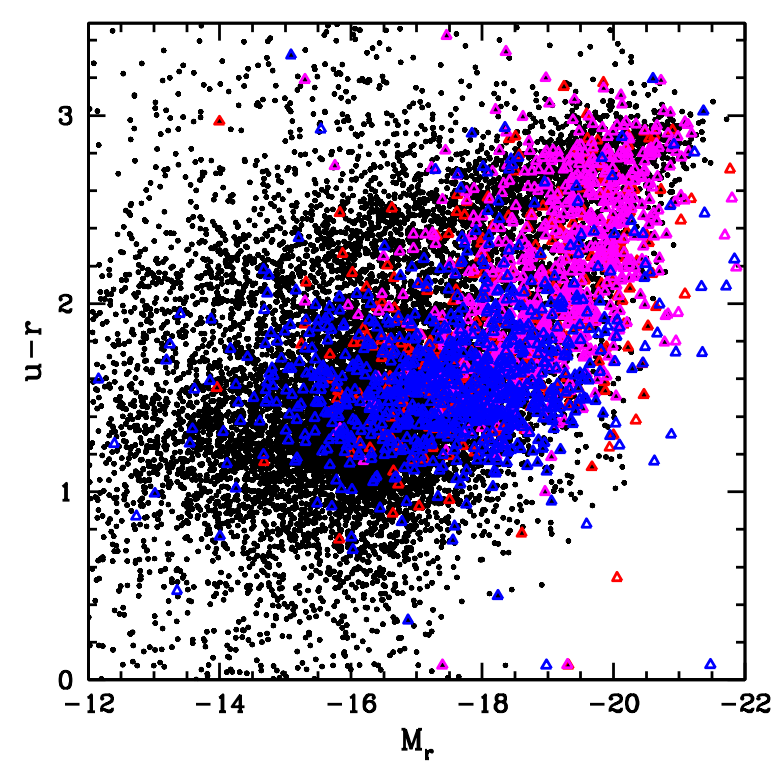

Fig. 2. $-M_{r}$ versus $u-r$ plot showing the distribution of nearby galaxies $(z<0.02)$ in KIAS VAGC. We designate spiral galaxies with arm classes by colored triangles: G (red), M (magenta) and F (blue).

nearby galaxies. In the figure, 1912 spiral galaxies are denoted as open triangles with colors coded by their arm classes; G (grand design, red), M (multiplearm, magenta) and $\mathrm{F}$ (flocculent, blue). The arm classes were based on the arm classification system of Elmegreen \& Elmegreen (1987). However, Ann \& Lee (2013) used three broad arm classes, grand design (G), multiple-arm (M), and flocculent $(\mathrm{F})$, to suppress the statistical noise in the correlation studies between the arm classes and other galaxy properties. Following the arm class definition of Elmegreen \& Elmegreen (1987), Ann \& Lee (2013) assigned AC 1-4 to flocculent arms, AC 5-9 to multiple arms, and AC 12 to grand design arms.

\section{LOCAL BACKGROUND DENSITY AS A MEASURE OF ENVIRONMENT}

The local background density is one of the most widely used parameters to represent the environment of a galaxy since Dressler (1980) determined the MDR for cluster galaxies. There are several ways to define the local background density. If we know the distances of the galaxies accurately enough to determine the threedimensional spatial distribution of galaxies, we can calculate the spatial density $\rho(r)$. However, except for the galaxies for which distances are determined by the Cepheid $P-L$ relation or Tully-Fisher relation (Tully \& Fisher 1977), the distances of galaxies are determined from the galaxy redshifts. Because the redshifts of galaxies are affected by the peculiar motions of galaxies due to the interactions with neighbor galaxies or large scale structures, the distances derived from the galaxy redshifts are not accurate enough, especially for galaxies in the local volume. To alleviate the effects of the inaccurate distances on the derivation of the local background density, it is common practice to derive a surface density $\Sigma$ centered on a target galaxy using the projected distance $r_{p}$ to neighbor galaxies, which is calculated from the distance of the target galaxy and angular separation between the target galaxy and its neighbors.

There are two practical methods to calculate the surface density $\Sigma$ using $r_{p}$ : one involves the use of fixed apertures and the other involves the use of projected distances to the $n$th nearest neighbor galaxy. The fixed aperture method with an aperture size of $5 \sim 8 h^{-1} \mathrm{Mpc}$ is known to be effective for densities at the superhalo scales, while the $n$th nearest neighbor method with small $n$ is best suited for densities inside high mass halos (Muldrew et al. 2012). Hence, we adopt the $n$th nearest neighbor method to derive the local background density in the present study. The number of neighbors to be searched, $n$, can be selected by considering the length scales relevant to the systems under investigation. A small $n$ such as $n=3$ or $n=5$ is suitable for the local environment of target galaxies in isolation or those in galactic groups, while a large $n$ such as $n \geq 9$ is suitable for the scale of clusters of galaxies. However, even if $n$ or an aperture is selected, the local background density of a galaxy cannot be determined uniquely because there are two free parameters left to be determined: the limiting absolute magnitude of the neighbor galaxies to be searched, $M^{*}$, and the maximum velocity difference of the neighbor galaxies, $\Delta V^{*}$.

\subsection{Effect of $M_{r}{ }^{*}$ and $\Delta V^{*}$ on Neighbor Search}

One of the most direct measurements of the galaxy environment is the projected distance to the nearest neighbor $\left(r_{p}\right)$. The morphology conformity between nearest neighbors in galactic satellite systems (Ann, Park, \& Choi 2008) and in field galaxies (Park, Gott, \& Choi 2008) strongly depends on $r_{p}$.

All galaxy environment measures are required to define neighbors of a target galaxy by constraining $M^{*}$ and $\Delta V^{*}$. Since the projected distance $\left(r_{p}\right)$ to the nearest neighbor is the most direct measure of the galaxy environment, we examined the dependence of the mean $r_{p}$ on $M^{*}$ and $\Delta V^{*}$ using nearby galaxies $(z<0.02)$ listed in the KISG VAGC. We varied $\Delta V^{*}$ from 300 to $1000 \mathrm{~km} / \mathrm{s}$ with several $M^{*}$. The results of some typical cases are shown Fig. 3. As shown in Fig. 3, the mean $r_{p}$ to the nearest galaxy increases from the neighbor galaxies selected using a faint $M^{*}$ to those selected by bright $M^{*}$. This is because the luminosity function of local galaxies increases from bright galaxies to faint ones in the luminosity ranges explored in Fig. 3. Because $M^{*}=-16.1$ corresponds to the SDSS observation limit of spectroscopic target galaxies at $z=0.02$, 


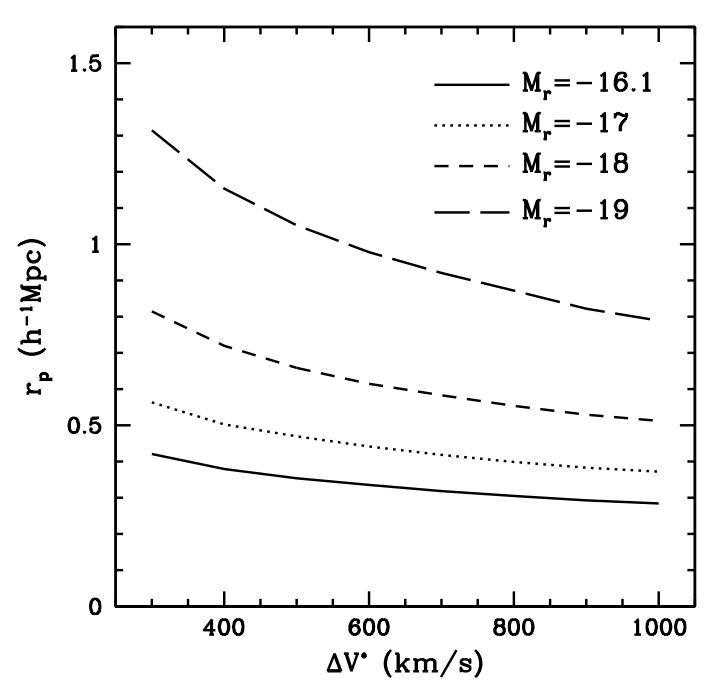

Fig. 3.- Projected distance to the nearest neighbor $r_{p}$ as a function of $\Delta V^{*}$. The line types distinguish the luminosity constraints: $M^{*}=-16.1$ (solid line), $M^{*}=-17($ dotted line), $M^{*}=-18$ (short dashed line), and $M^{*}=-19$ (long dashed line).

the mean projected separation of galaxies in the present volume limited sample is $\sim 0.35 h^{-1} \mathrm{Mpc}$.

For a given $M^{*}$, the mean $r_{p}$ decreases with increasing $\Delta V^{*}$, regardless of $M^{*}$. However, the slopes of the variations are much different. For the 300 to $1000 \mathrm{~km} / \mathrm{s}$ $\Delta V^{*}$ range, the mean $r_{p}$ changes from $\sim 0.3$ to $\sim 0.4$ $h^{-1} \mathrm{Mpc}$ for $M^{*}=-16.1$, while it changes from $\sim 0.8$ to $\sim 1.3 h^{-1} \mathrm{Mpc}$ for $M^{*}=-19$. The small changes in $r_{p}$ for the galaxies selected by $M^{*}=-16.1$ is due to presence of neighbor galaxies with $\Delta V$ smaller than $300 \mathrm{~km} / \mathrm{s}$ within $r_{p} \approx 0.4 h^{-1} \mathrm{Mpc}$. The large $r_{p}$ for galaxies brighter than $M_{r}^{*}=-18$ with $\Delta V$ less than $300 \mathrm{~km} / \mathrm{s}$ is due to the absence of bright companions as the closest neighbor. The mean $r_{p}$ of $\sim 1 h^{-1} \mathrm{Mpc}$ is about three times larger than the virial radius of a bright spiral galaxy such as the Milky Way.

Fig. 4 shows the mean $r_{p}$ of the $n$th nearest neighbor as a function of $\Delta V^{*}$ for $M^{*}=-16.1$ and $M^{*}=-18$. Here, we show three cases of $n=3, n=7$, and $n=21$. As shown in Fig. 4, the mean $r_{p}$ of the 3rd nearest neighbor with $M^{*}=-16.1$ increases very slowly from $\sim 0.5 h^{-1} \mathrm{Mpc}$ for $\Delta V^{*}=1000 \mathrm{~km} / \mathrm{s}$ to $\sim 1 h^{-1} \mathrm{Mpc}$ for $\Delta V^{*}=300 \mathrm{~km} / \mathrm{s}$. The mean $r_{p}$ of the $3 \mathrm{rd}$ nearest neighbor with $M^{*}=-18$ is very similar to the mean $r_{p}$ of the 7 th nearest neighbor with $M^{*}=-16.1$. They vary slowly from $\sim 1 h^{-1} \mathrm{Mpc}$ to $\sim 1.3 h^{-1} \mathrm{Mpc}$ for $\Delta V^{*}$ between 1000 and $500 \mathrm{~km} / \mathrm{s}$ and becomes $\sim 1.8$ $h^{-1} \mathrm{Mpc}$ for $\Delta V^{*}=300 \mathrm{~km} / \mathrm{s}$. A similar trend of rapid increase of $r_{p}$ for $\Delta V^{*} \lesssim 500 \mathrm{~km} / \mathrm{s}$ is observed for other values of $n$. Thus, there appears to be a common property, probably caused by the paucity of companions for a small $\Delta V$. This suggests that a $\Delta V^{*}$ of $\sim 400 \mathrm{~km} / \mathrm{s}$ is a lower limit for searching neighbor galaxies, regardless of the length scales to be studied.

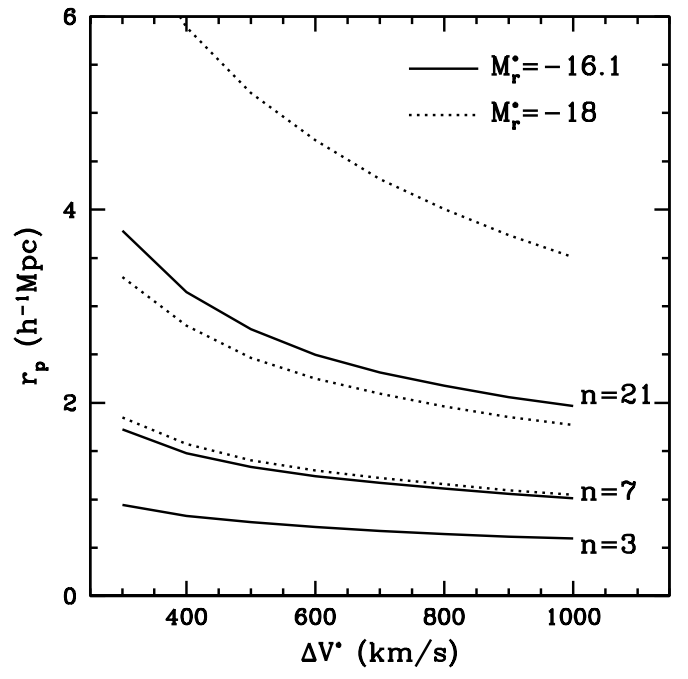

Fig. 4. - Projected distance to the $n$th nearest neighbor $r_{p}$ as a function of $\Delta V^{*}$. The line types distinguish the luminosity constraint: $M^{*}=-16.1$ (solid line) and $M^{*}=$ -18 (dotted line). The number of nearest neighbors $n$ is indicated for the case of $M^{*}=-16.1$.

Since $r_{p}$ characterizes the length scales relevant to the local background densities, the environmental properties represented by the local background densities derived from the 3rd nearest neighbor with $M^{*}=$ -18 are equally well described by those from the 7 th nearest neighbor with $M^{*}=-16.1$. The same is true for the mean $r_{p}$ derived by $n=21$ with $M^{*}=-16.1$ and $n=7$ with $M^{*}=-18$. Because we used local background densities normalized by their mean values, the frequency distributions of the local background densities derived from the two methods are expected to be nearly identical. In the literature (Muldrew et al. 2012 , references are there in), several values of $n$ were used to derive local background densities. However, as seen in Fig. 4, a large $n$ such as $n=21$ should be used for the structures with length scales larger than $\sim 4 h^{-1} \mathrm{Mpc}$, while a small $n$ such as $n=3$ appears to be suitable for systems smaller than $\sim 1 h^{-1} \mathrm{Mpc}$. For small systems such as galactic satellite systems or group of galaxies, $n \leq 5$ with $\Delta V^{*} \approx 500 \mathrm{~km} / \mathrm{s}$ appears to be a better choice for the local background densities.

\subsection{Local Background Density from the $n$th Nearest Neighbor}

Currently, the $n$th nearest neighbor technique is most widely used method to derive the local background density. It has been applied to nearby galaxies as well as to large scale structures. The local background density obtained using the $n$th nearest neighbor is defined as

$$
\Sigma_{n}=\frac{n}{4 \pi r_{p, n^{2}}},
$$




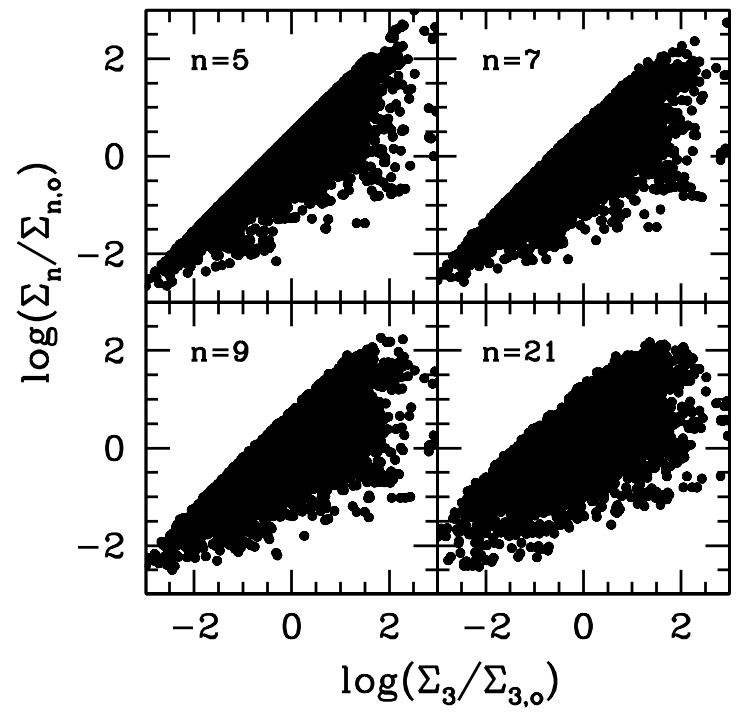

Fig. 5. - Correlation between background densities derived from the $n$th nearest neighbor method. We selected densities from $n=3$ as the reference densities. We fixed the two searching parameters: $\Delta V^{*}=500 \mathrm{~km} / \mathrm{s}$ and $M^{*}=-16.1$.

where $r_{p, n}$ is the projected distance to the $n$th nearest galaxy that is brighter than $M^{*}$ with $|\Delta V|<\Delta V^{*}$. We use $\Delta V^{*}=500 \mathrm{~km} / \mathrm{s}$ and $M^{*}=-16.1$ for the representative peculiar velocity and luminosity constraints. However, other values of $\Delta V^{*}$ and $M^{*}$ are used together to examine the dependence of the density distributions on $\Delta V^{*}$ and $M^{*}$. In the literature, as discussed by Muldrew et al. (2012), there is no consensus on the number of neighbor galaxies to be searched. It ranges from 3 to 60 with a preference for small $n$ values for local background density relevant to the fine morphologies of galaxies. A large $n$ such as $n=21$ appears to be a better measure of the local background density relevant to large scale structure (see Park, Gott, \& Choi 2008, for example). Fig. 5 shows the correlations between the densities derived from $n=5,7,9,21$ and that from $n=3$. We maintain the searching constraints $\Delta V^{*}$ and $M^{*}$ constant at $500 \mathrm{~km} / \mathrm{s}$ and -16.1 , respectively. There are fairly good correlations between the densities derived from different $n$, although the scatter is too large to be ignored. The scatter is mainly due to the presence of small scale regions with different densities $\Sigma_{3}$ within regions of a density $\Sigma_{n}$ for a large $n$. As expected from the dependence of scale lengths on $n$, the densities from $n=5$ exhibit the best correlation with $n=3$, while those from $n=21$ exhibit the worst correlation.

Fig. 6 shows the fractional distribution of densities derived from the $n$th nearest neighbor with $M^{*}=-16.1$ and -18 as well as $\Delta V^{*}=500$ and $1000 \mathrm{~km} / \mathrm{s}$. There is no significant difference between the fractional distributions for the same $n$. The density distribution

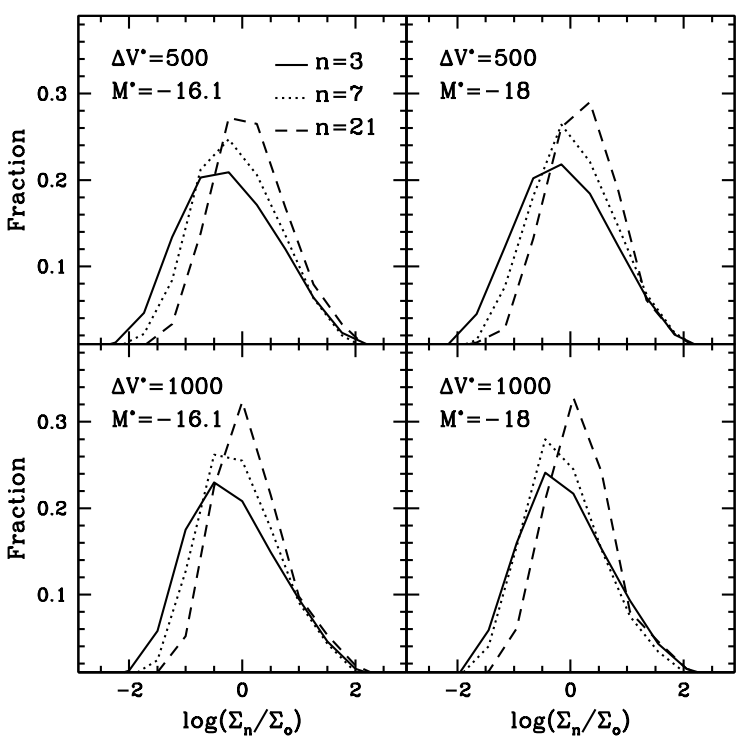

Fig. 6. - Fractional distribution of background densities obtained using the $n$th nearest neighbor. Left and right panels present the densities derived using $M^{*}=-16.1$ and $M^{*}=-18$, respectively.

pattern is less affected by $M^{*}$ than by $\Delta V^{*}$. However, the case for $n=3$ with $\Delta V^{*}=500 \mathrm{~km} / \mathrm{s}$ and $M^{*}=-16.1$ exhibits the largest density ranges, which results in the highest resolution. For the same $\Delta V^{*}$ and $M^{*}$, the density ranges decrease from the smallest to the largest $n$ with a peak density shift towards higher densities. The shift in the peak density with larger $n$ is due to the larger contribution of dense regions such as clusters of galaxies for larger $n$. The dependence of density resolution on $n$ is due to the characteristic scales associated with the derived densities. As demonstrated in Fig. 4, the mean $r_{p}$ depends on $n$ if we fix $M^{*}$ and $\Delta V^{*}$. When compared with the densities from the fixed aperture method, the ranges of the densities derived from the $n$th nearest neighbor are larger than those from the fixed apertures. This suggests that if morphological features such as spiral arm classes are mostly affected by small scale perturbations, the most relevant density estimator would be the one derived from the $3 \mathrm{rd}$ nearest neighbor with $\Delta V^{*}=500 \mathrm{~km} / \mathrm{s}$.

\subsection{Disk Morphology and Local Background Densities}

The arm classes, Hubble type, and bar type fractions of 1912 spiral galaxies as a function of local background densities are shown in Figs. 7, 8 and 9. The local background densities in Figs. 7 and 9 were derived using $n=3$, while in Fig. 8 they were derived using $n=5$. We use $\Delta V^{*}=500 \mathrm{~km} / \mathrm{s}$ in all the cases except in the lower panels of Fig. 7 where $\Delta V^{*}=1000 \mathrm{~km} / \mathrm{s}$. We used the same luminosity constraint of $M^{*}=-16.1$ in Figs. 7 and 8; however, we used $M^{*}=-18$ in Fig. 9 to ob- 


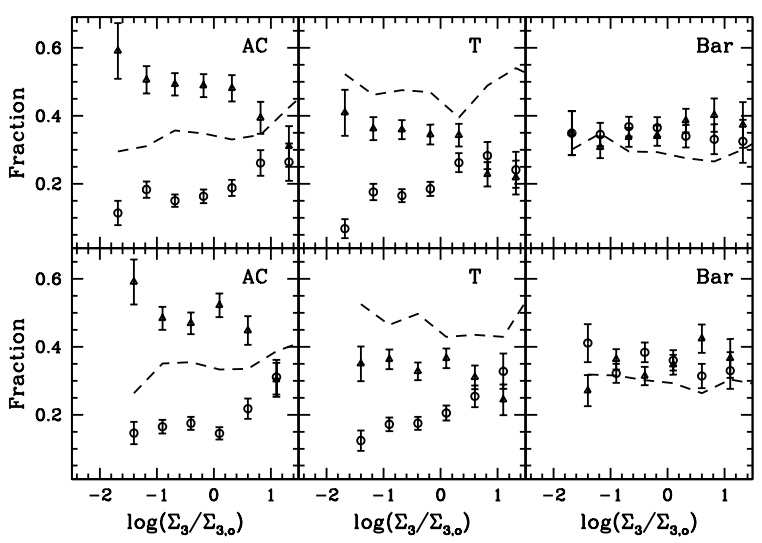

Fig. 7.- Fraction of disk morphology as a function of the local background density $\left(\Sigma_{3}\right)$ derived from $\Delta V^{*}=500 \mathrm{~km} / \mathrm{s}$ (upper panels) and $\Delta V^{*}=1000 \mathrm{~km} / \mathrm{s}$ (lower panels). We fixed $M^{*}$ to $M_{r}=-16.1$. The fractions of the arm class, Hubble type, and bar type are plotted in the left, middle, and right panels, respectively. The open circles with error bars represent the grand design in the left panels, early-type spirals in the middle panels, and SA in the right panels. The filled triangles with error bars represent the flocculent arms in the left panels, late-type spirals in the middle panels, and SB spirals in right panels. The short dashed lines represent multiple-arm in the left panels, intermediate-types in the middle panels, and $\mathrm{SAB}$ in the right panels.

serve the effects of the luminosity constraint. We used the arm class, Hubble type, and bar type of 1912 spiral galaxies from the visual classification of Ann \& Lee (2013). There is a strong dependence of arm class and Hubble type on the local background densities; however, the bar type exhibits a very weak dependence on the local background densities.

As shown in the left panels of Figs. 7, 8, and 9, the fraction of grand design galaxies (open circles with error bars) steadily increases from low density regions to high density regions, while that of flocculent galaxies (filled triangles with error bars) decreases. Since structural properties are very different, the opposite trends in grand design spirals and flocculent spirals demonstrates the dependence of arm class on the local background density. The fraction of multiple-arm galaxies (dashed lines), which have intermediate arm properties between those of grand design and flocculent arms, is nearly constant along the local background density. The correlations between arm class fraction and local background densities is not affected much by the neighbor search constraint $\Delta V^{*}$ for $n=3$ and $n=5$ when we use the luminosity constraint $M^{*}$ as the limiting magnitude of the volume limited sample. However, for the case of $M^{*}=-18$, the correlation becomes too weak to be easily recognized. The clearest correlation is observed for the local background densities derived from $n=3$ with $\Delta V^{*}=500 \mathrm{~km} / \mathrm{s}$ and $M^{*}=-16.1$.

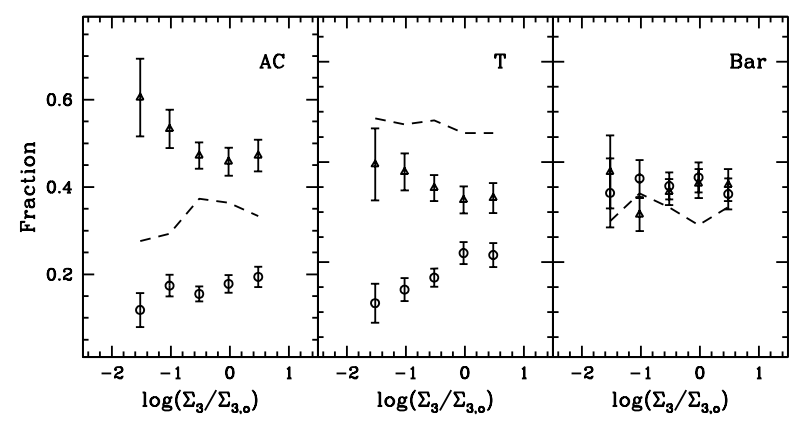

Fig. 8. - Fraction of disk morphology as a function of local background density $\left(\Sigma_{5}\right)$ derived from $\Delta V^{*}=500 \mathrm{~km} / \mathrm{s}$. We fixed $M^{*}$ as $M_{r}=-16.1$. The meanings of symbols and line types are the same as those of Fig. 7 .

For the Hubble types plotted in the middle panels of Figs. 7, 8, and 9, the fractions of early-type spirals (open circles with error bars) increase with increasing local background density, while those of latetype spirals (filled triangles with error bars) decrease with increasing local background density. The correlation of the early-type fractions with local background density is stronger than that of the grand design fractions. However, the anti-correlation of the late fractions with local background density is weaker than that of the flocculent arms, especially for the densities with $\Delta V^{*}=1000 \mathrm{~km} / \mathrm{s}$, regardless of $n$ and $M^{*}$. Because intermediate-type spirals (dashed lines) have intermediate morphological properties between those of earlyand late-type spirals, we expect that the correlation between their fractions and local background densities are also intermediate; in other words, we expect almost no correlation. In this regard, the nearly constant fractions of intermediate-type spirals, shown in the upper middle panel in Fig. 7 and middle panel in Fig. 8, appear to be consistent with the general MDR. The decreasing trend of intermediate spiral fractions shown in the lower panels of Fig. 7 appears plausible because the late-type fractions also decrease with density at a similar rate. For the densities from $n=3$ and $M^{*}=-18$ shown in Fig. 9, the correlations between the disk morphology and $\Sigma_{3}$ with $M^{*}=-18$ are much weaker than those shown in Figs. 7 and 8.

Ann \& Lee (2013) determined the dependence of the disk morphology on the local background density, calculated using the nearest neighbor method with $n=5$. Here, we present the same dependence with $n=3$ (Fig. 7) because the local background density derived from $n=3$ exhibits the highest resolution owing to its large density range (Fig. 6). As shown in the left panel of Fig. 7, the fractions of arm classes are highly dependent on the local background density. The fraction of flocculent galaxies (filled triangles) is $\sim 0.6$ at $\log \left(\Sigma_{3} / \Sigma_{3,0}\right)=-2$ and decreases monotonically as the local background density increases, reaching $\sim 0.3$ at $\log \left(\Sigma_{3} / \Sigma_{3,0}\right)=1$. Conversely, the fraction of grand 


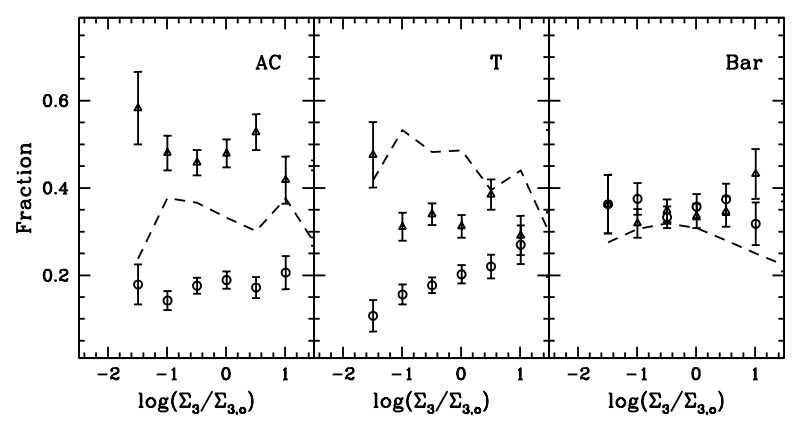

Fig. 9.- Fraction of disk morphology as a function of the local background density $\left(\Sigma_{3}\right)$ derived from $\Delta V^{*}=500 \mathrm{~km} / \mathrm{s}$. We fixed $M^{*}$ as $M_{r}=-18$. The fractions of arm class, Hubble type, and bar type are plotted in the left, middle, and right panels, respectively. The meanings of symbols and line types are the same as those of Fig. 7.

design galaxies (open circles) increases nearly monotonically from $\sim 0.1$ at $\log \left(\Sigma_{3} / \Sigma_{3,0}\right)=-2$ to $\sim 0.35$ at $\log \left(\Sigma_{3} / \Sigma_{3,0}\right)=1$. Multiple-arm galaxies (dashed line) exhibit an increasing trend similar to that of grand design galaxies, but with a much smaller gradient. The general trend of the dependence of arm class on the local background density shown in Fig. 7 agrees well with that given in Ann \& Lee (2013), but there is a slight difference between them because of the different density resolution resulting from the different $n$ used. The dependence of the Hubble type on the local background density is stronger than that of the arm class. This is because the bulge-to-disk ratio, which is strongly correlated with the Hubble type, is determined during the galaxy formation process with a large bulge-to-disk ratio in high density regions following the MDR. Conversely, the arm class is mainly determined by the mode of the disk instability, which depends on the external tidal perturbation as well as the disk mass relative to halo mass.

\section{OTHER MEASURES OF LOCAL ENVI- RONMENT}

\subsection{Projected Distance to the Nearest Neigh- bor}

The projected distance $\left(r_{p}\right)$ appears to be a good measure for the galaxy environment because the morphology of a galaxy is mostly affected by its neighbor galaxies (Park, Gott, \& Choi 2008). The strong dependence of morphology conformity between a host galaxy and its satellite galaxies (Weinmann \& van den Bosch 2006; Ann, Park, \& Choi 2008) is a good example of the role $r_{p}$ has on the galaxy morphology. Sometimes, $r_{p}$ is normalized by the virial radius of the neighbor galaxy because the morphology transformation driven by interactions with neighbor galaxies is effective when galaxies are located within the virial radii

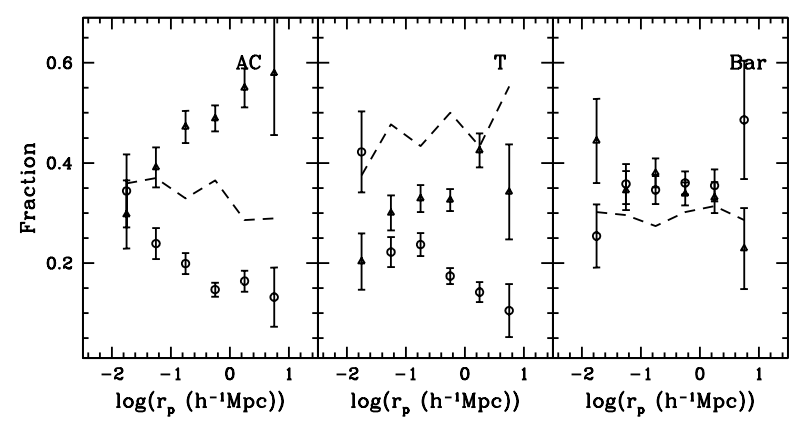

Fig. 10. - Arm class, Hubble type, and bar type fractions as functions of $r_{p}$ with $M_{r}=-18$. The meanings of symbols and line types are the same as those of Fig. 7.

of their neighbor galaxy (Park \& Choi 2009).

Fig. 10 shows the arm class, Hubble type, and bar type fractions as functions of $r_{p}$; these were derived with $\Delta V^{*}=500 \mathrm{~km} / \mathrm{s}$ and $M^{*}=-18$. It is apparent that the arm class, Hubble type, and bar type do depend on $r_{p}$. The fraction of grand design spirals decreases with increasing $r_{p}$, while flocculent spirals exhibit the opposite trend. The fraction of multiple-arm spirals is nearly constant until $r_{p}=1 \quad h^{-1} \mathrm{Mpc}$ and decreases thereafter. For the Hubble type case, the fraction of early-type spirals decreases with $r_{p}$ and that of latetype spirals increases with $r_{p}$. Meanwhile, the bar type also exhibits a clear dependence on $r_{p}$, in the sense that the SA fraction increases with increasing $r_{p}$ and the SB fraction decreases with $r_{p}$, while the SAB exhibits a nearly constant fraction along $r_{p}$. Thus, $r_{p}$ appears to be a better measure of the local environment to distinguish the disk morphology, especially arm classes, than the local background density.

Correlations between disk morphology and $r_{p}$ are stronger than those between disk morphology and local background density (Figs. 7 and 8). In particular, $r_{p}$ exhibits much stronger correlations with grand design spirals and early-type spirals than for the local background densities (Figs. 7 and 8). However, if we use $r_{p}$ derived with $M_{r}=-16.1$, correlations become much weaker, as shown in Fig. 11. The reason for stronger correlations for $r_{p}$ with $M_{r}=-18$ is the dependence of the interaction strength on the neighbor luminosity, i.e., mass. This explanation is supported by the strongest dependence of disk morphology on the tidal index $(T I)$ shown in Fig. 13.

Fig. 12 shows the dependence of the disk morphology on the projected distance normalized by the nearest neighbor's virial radius $\left(r_{\text {virnei }}\right), r_{p} / r_{\text {virnei }}$. The general dependence is similar to that shown in Fig. 10 However, the arm class exhibits a better correlation with $r_{p}$ than $r_{p} / r_{v i r n e i}$, while the Hubble type exhibits a better correlation with $r_{p} / r_{\text {virnei }}$ because there is a break in the grand design fraction at small normalized projected separations of $r_{p} / r_{\text {virnei }} \approx 0.1$. Meanwhile, a 


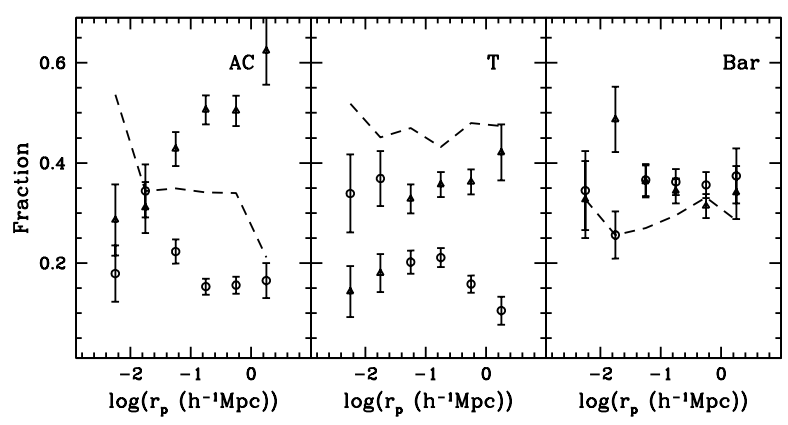

Fig. 11. - The arm class, Hubble type, and bar type fractions as functions of $r_{p}$ with $M_{r}=-16.1$. The arm class, Hubble type, and bar type are plotted in the left, middle, and right panels, respectively. The meanings of symbols and line types are the same as those of Fig. 7 .

similar break occurs in the early-type fractions at small projected separations of $r_{p} \approx 0.1 h^{-1} M p c$. The grand design fraction is nearly constant at $r_{p}>1 h^{-1} M p c$ or $r_{p} / r_{\text {virnei }}>1$. The presence of a break in the grand design fraction at $r_{p} / r_{\text {virnei }} \approx 0.1$ and the absence of a break in the early-type fraction at the same $r_{p} / r_{\text {virnei }}$ suggest that the disk instability is affected more by the close neighbors than the global structure of disk, which is represented by the bulge-to-disk ratio. The nearly constant fraction of the grand design spirals at $r_{p} / r_{\text {virnei }} \approx 10$ implies that the length scales on which the arm class depends are smaller than those of the Hubble type because early-type fractions exhibit a nearly monotonic decrease to $r_{p} / r_{\text {virnei }} \approx 10$. There is little difference in the dependence of bar fractions between $r_{p}$ and $r_{p} / r_{v i r n e i}$.

\subsection{Tidal Index}

Tidal interactions with neighbor galaxies are considered to be the main drivers of morphology change of a galaxy. A strong correlation of disk morphology with the local background density suggests a parameter that represents the tidal strength of neighboring galaxies can be used as a measure of the local environment of galaxies.

Karachentsev \& Makarov (1999) introduced the TI as a measure of the tidal interaction among galaxies in the local volume $(D<10 \mathrm{Mpc})$. They used the threedimensional separation between neighboring galaxies based on the distances, most of which are determined by direct methods such as photometric distances or group membership with known group distances. However, we cannot use the three-dimensional separation of neighboring galaxies owing to the large uncertainties that are inherent in the kinematic distances derived from the galaxy redshifts. We defined a TI similar to Karachentsev \& Makarov (1999) using the projected separation between a target galaxy $i$ and its neighbor galaxies as

$$
T I_{i}=\max \left[\log \left(\frac{G m_{k}}{r_{i k}^{3}}\right)+C\right], k=1,2,3, \ldots . N
$$

where $m_{k}$ and $r_{i k}$ are, respectively, the mass and projected separation between the target galaxy $i$ and neighbor galaxy $k$. $C$ is the normalization constant, which corresponds to the TI using a galaxy containing the Milky Way mass at a separation of $1 h^{-1} \mathrm{Mpc}$. We used galaxy masses determined from $M_{r}$ using a mass-to-light ratio of 1 .

Fig. 13 shows the dependence of disk morphology on the $T I$. Here, we used the neighbor search constraints $\Delta V^{*}=500 \mathrm{~km} / \mathrm{s}$ and $M^{*}=-16.1$. As expected, the arm class and Hubble type clearly depend on $T I$. The fractions of grand design and multiple-arm spirals increase with $T I$, while that of the flocculent spirals decreases with $T I$. This means that strong tidal interactions are likely to drive a large scale disk instability, which leads to generation of global density waves. However, this general trend is kept at moderate ranges of $T I$. The flat distribution of the grand design fractions at $T I<0$ suggests that the tidal strength weaker than that caused by a galaxy with a Milky way mass at a separation of $\sim 1 h^{-1} \mathrm{Mpc}$ does not generate global density waves to drive grand design arms. The grand design fraction also decreases at extremely strong tidal forces, $T I>5$. This is due to the formation of a single massive arm or chaotic arms, which are classified as flocculent spirals. For the Hubble type case, the fraction of early-type spirals increases with $T I$, while that of late type spirals decreases with $T I$ and exhibit a bump at $T I \approx 3$. Meanwhile, the intermediate-type spirals exhibit nearly constant fractions.

Contrary to the cases for the local background density and projected distance to the nearest neighbor, the velocity constraint of neighbor galaxies is much tighter. As shown in Fig. 14 in which we derived TI with $\Delta V^{*}=1000 \mathrm{~km} / \mathrm{s}$ and $M^{*}=-16.1$, the dependence of

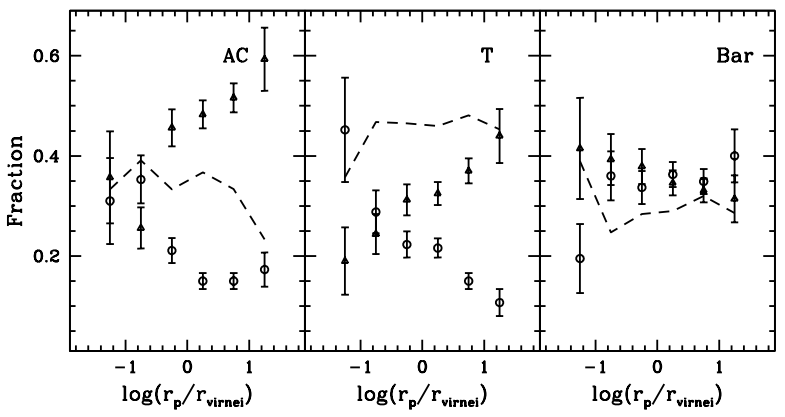

Fig. 12. - The arm class, Hubble type, and bar type fractions as functions of $r_{p} / r_{v i r n e i}$. The arm class, Hubble type, and bar types are plotted in the left, middle, and right, respectively. The meanings of symbols and line types are the same as those of Fig. 7. 


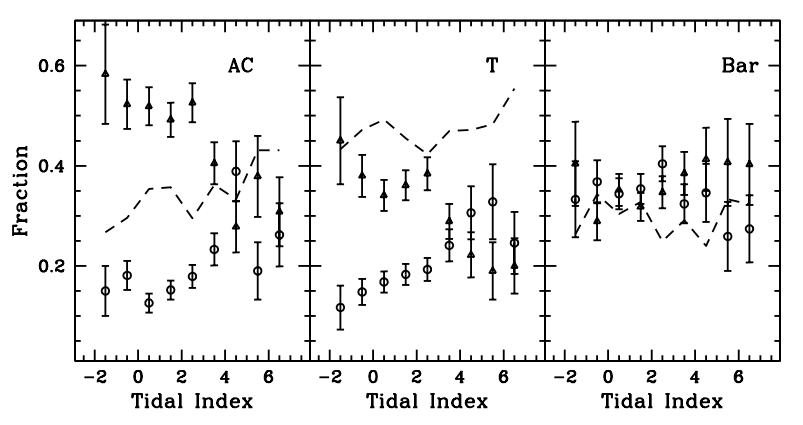

Fig. 13. - The arm class, Hubble type, and bar type fractions as functions of $T I$. Here, $T I$ is derived with $\Delta V^{*}=500 \mathrm{~km} / \mathrm{s}$. The arm class, Hubble type, and bar types are plotted in the left, middle, and right panels, respectively. The meanings of symbols and line types are the same as those of Fig. 8.

disk morphology on $T I$ is much weaker than that in Fig. 13. In particular, there appears to be no correlation between the arm class and $T I$ for $T I<4$. A similar trend is observed for the Hubble type with $T I<4$. This suggests that $\Delta V^{*}=1000 \mathrm{~km} / \mathrm{s}$ allows too many interlopers in the neighbor galaxies.

\section{DISCUSSION}

We explored three measures of local environment: local background density $\left(\Sigma_{n}\right)$, projected distance to the nearest neighbor $\left(r_{p}\right)$, and $T I$ which quantifies the maximum tidal strength from neighbor galaxies. These measures exhibit fairly good correlations with disk morphology characterized by arm class (G, M, F), Hubble type (early, intermediate, late) and bar type (SA, SAB, SB). However, the environment measures are heavily affected by the neighbor search parameters $\left(M^{*}\right.$ and $\left.\Delta V^{*}\right)$ that constrain the neighbor's luminosity and velocity relative to target galaxy.

It is natural to take $M^{*}$ as the limiting luminosity that defines the present sample of galaxies as a volume limited sample, i.e., $M^{*}=-16.1$. However, we examined other values of $M^{*}$. As expected, we found the best correlations between the disk morphology and environment measures $(\Sigma$ and $T I)$ when we used $M^{*}=-16.1$; however, $M^{*}=-18$ is better for $r_{p}$. In particular, the arm class exhibited the best correlation with $r_{p}$ when it is derived with $M^{*}=-18$. There is no preference for $\Delta V^{*}$ because the particular velocities, which determine $\Delta V^{*}$, depend on the environment itself. Too small values of $\Delta V^{*}$ cause a large fraction of neighbors to be missed, while too large values of $\Delta V^{*}$ introduce a large number of interlopers that degrade the correlation between the disk morphology and environment. However, as shown in Fig. 3, the dependence of $r_{p}$ on $\Delta V^{*}$ is very weak, especially for $M^{*}=-16.1$. We extensively examined two cases of $\Delta V^{*}: 500$ and $1000 \mathrm{~km} / \mathrm{s}$. The $500 \mathrm{~km} / \mathrm{s}$ value of $\Delta V^{*}$ is widely used to search

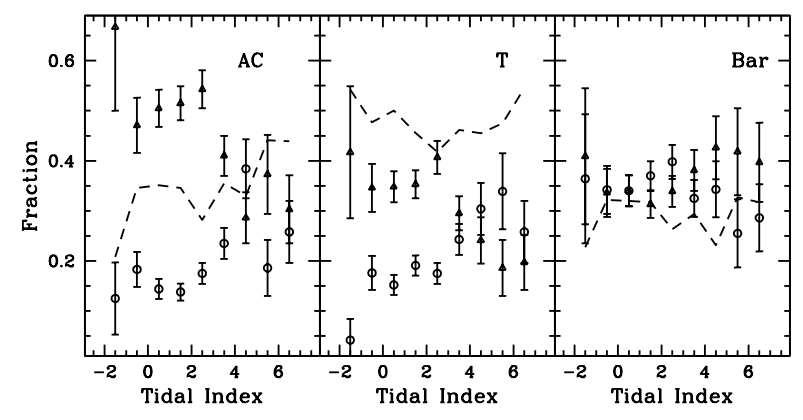

Fig. 14. - The arm class, Hubble type, and bar type fractions as functions of $T I$. Here, $T I$ is derived using $\Delta V^{*}=1000 \mathrm{~km} / \mathrm{s}$. The arm class, Hubble type, and bar types are plotted in the left, middle, and right panels, respectively. The meanings of symbols and line types are the same as those of Fig. 8.

for nearby neighbors such as satellite galaxies (for example see Ann, Park, \& Choi (2008)). It is similar to those used by Park \& Choi (2009) to search for the nearest neighbor. $\Delta V^{*}$ values used by Park \& Choi (2009) were $400 \mathrm{~km} / \mathrm{s}$ for neighbors of spiral galaxies and $600 \mathrm{~km} / \mathrm{s}$ for neighbors of elliptical galaxies. The $600 \mathrm{~km} / \mathrm{s}$ value of $\Delta V^{*}$ is a typical value for selecting isolated galaxies; it was used in, for instance, Park \& Choi (2009). We found that better correlations between the disk morphology and measures of local environment are observed when we use $\Delta V^{*}=500 \mathrm{~km} / \mathrm{s}$. However, as demonstrated in Fig. 7, correlations between the disk morphology and $\Sigma_{n}$ are not affected much by $\Delta V^{*}$ for small $n$ values such as $n=3$ or $n=5$. The preference for a $\Delta V^{*}$ value of $500 \mathrm{~km} / \mathrm{s}$ is more pronounced in the dependence of disk morphology on $T I$, which is thought to be a direct measure of tidal strength of neighboring galaxies. Tighter constraints on $|\Delta V|$ results in a better correlation between the disk morphology and environment measures because interlopers are suppressed.

Arm classes exhibit stronger dependencies on $r_{p}$ than $\Sigma$ and $T I$, while Hubble types exhibit stronger dependencies on $\Sigma$. The strong dependence of the arm class on $r_{p}$ is due to the close relationship between the arm class and mode of disk instability, which is thought to be determined primarily by the perturbations imposed by the nearest neighbor. The less clear correlation between the arm class and $\Sigma$ could be due to multiple criteria of the arm class (continuity, symmetry, and length) that degrade correlations. Some arms are classified as a flocculent galaxy owing to the lack of symmetry but have continuity, while other arms are classified as a flocculent galaxy owing to the fleece-like structure. A galaxy with two symmetric arms can be classified as a grand design galaxy if there is no other structure, but it can be classified as a multiple-arm galaxy if there is an outer ring or a pseudo-ring. Thus, the arm class of multiple arms can be merged with grand design arms 
if we consider only symmetry and continuity. On the contrary, the Hubble type subdivision of spiral galaxies, i.e., Hubble stage, is basically classified by a single parameter, bulge-to-disk ratio, which obeys the MDR.

It is also possible that the correlation between the Hubble type and local background density is more fundamental than that between the arm class and local background density. The Hubble type, which is distinguished by the bulge-to-disk ratio, is determined during the formation processes of the bulge and disk, while the arm class, which is distinguished by the spiral arm structures and determined by disk instability, is set up after the disk forms. As dictated by MDR (Dressler 1980), which is closely related with the morphology-luminosity relation (MLR) of galaxies (Park et al. 2007), bulge dominated galaxies are likely to be formed in dense regions. An elliptical galaxy is an extreme case of a bulge dominated galaxy. The bulge of the early-type spiral is also thought to be formed in dense regions where a rapid collapse of the central region is likely to occur before the disk forms. However, in less dense regions, the central regions slowly collapse owing to the dependence of the dynamical time scale on the density, $\tau \sim(1 / G \rho)^{1 / 2}$. Thus, it appears that it is better to use the bulge-to-disk ratio as the primary parameter to distinguish the Hubble stage. It can be supported by the fact that the openness of the spiral arm depends on the bulge mass. Large bulges drive tightly wound spiral arms, while small bulges drive loosely wound spiral arms.

It seems of interest to clarify whether the bar type depends on the local environment. There have been many investigations regarding the relation between the bar fraction and environment (see Lee et al. (2012) and references therein). The nature versus nurture controversy for bars in disk galaxies is one of the long-lasting issues in galaxy evolution. The present analysis does not yield a clear conclusion, but we do obtain some insights. As shown in Figs. 7, 8, and 9, there is no strong bar type dependence on the local background density, especially in the galaxies located in the moderate density regions. However, there is a weak trend of increase in the fraction of SB galaxies with increasing density and decrease in the fraction of SA galaxies with increasing density (upper right panel of Fig. 7) for $\Sigma_{3}$, especially those derived from $\Delta V^{*}=500 \mathrm{~km} / \mathrm{s}$. Since $\Sigma_{3}$ with $\Delta V^{*}=500 \mathrm{~km} / \mathrm{s}$ and $M^{*}=-16.1$ provide a high density resolution and strong correlations with the arm class and Hubble type, it seems plausible that the unsolved issue of the nature versus nurture controversy is partly due to a lack of density resolution required for a detailed analysis. Of course, it is also plausible that the recurrent formation and destruction of bars demolish the correlation between the bar fraction and environment. However, if bars are robust enough to survive the internal and external destruction mechanisms, the controversy may be settled if we devise a better measure of galaxy environment. This argument is supported by better correlations between the bar fraction and $T I$, which is the most direct measure of the tidal strength from neighboring galaxies.

\section{CONCLUSIONS}

It is apparent that the disk morphology is closely related with the local environment of galaxies. Arm class and Hubble type exhibit a clear dependence on three measures of the local environment: local background density $(\Sigma)$, projected distance to the nearest neighbor galaxy $\left(r_{p}\right)$, and tidal index $(T I)$. However, the correlation strength depends on the environmental measures used. Since measures of the galaxy environment are constrained by the neighbor search parameters, $M^{*}$ and $\Delta V^{*}$, correlation strengths also depend on $M^{*}$ and $\Delta V^{*}$. It was found that $\Delta V^{*}=500 \mathrm{~km} / \mathrm{s}$ is better than $\Delta V^{*}=1000 \mathrm{~km} / \mathrm{s}$ for all three measures, $\Sigma$, $r_{p}$, and $T I$, but $M^{*}$ can be different for the measures of the selected environment.

Arm class exhibited the strongest correlation with $r_{p}$ when $r_{p}$ is derived from the neighbor search constraints $M^{*}=-18$ and $\Delta V^{*}=500 \mathrm{~km} / \mathrm{s}$. The fraction of grand design spirals decreases with increasing $r_{p}$, while that of flocculent spirals increases with it. Multiple-arm spirals exhibit a nearly constant fraction for $r_{p}<1 h^{-1} \mathrm{Mpc}$ and decreases as $r_{p}$ increases further. $T I$ as well as $\Sigma_{n}$ with $n=3$ and 5 are also closely related with the arm classes of spiral galaxies; however, they exhibit a weaker correlation than $r_{p}$. It is worth noting that the luminosity constraint of $r_{p}$ that gives strongest correlation with arm class is $\sim 2$ magnitude brighter than the limiting magnitude that defines the present volume limited sample, $M^{*}=-16.1$. This means that the arm class is mostly affected by a massive neighbor galaxy, which is close enough to significantly perturb the disk instability of target galaxy.

The local background density, $\Sigma_{n}$, is most closely related with the Hubble stage of spiral galaxies; however, $r_{p}$ and $T I$ also exhibit fairly good correlations with Hubble type. The best correlation was found for $\Sigma_{5}$ with $M^{*}=-16.1$ and $\Delta V^{*}=500 \mathrm{~km} / \mathrm{s}$. The fraction of early-type spirals increases with increasing $\Sigma$ and $T I$ as well as decreasing $r_{p}$, while that of latetype spirals exhibited opposite trends. Intermediatetype spirals exhibited a nearly constant fraction along $\Sigma, T I$, and $r_{p}$. The luminosity constraint of $r_{p}$ was $M^{*}=-18$, while that for $\Sigma$ and $T I$ was $M^{*}=-16$.

The bar type exhibited a weak dependence on the three environment measures, but there was a high frequency of strongly barred galaxies at high density regions where we expect relatively strong tidal strength and small separations of neighbor galaxies. The opposite is true for non-barred galaxies. Thus, the present results support the idea that bar formation is affected by the environment of galaxies.

The strong correlations between the frequencies of Hubble type of spiral galaxies, i.e., Hubble stage, and the local background density appear to originate from 
the MDR and MLR of galaxies, which govern the galaxy morphology throughout the galaxy formation process. This is the reason why the Hubble type exhibits a fairly strong correlation with $\Sigma$. The strong dependence of the frequencies of the arm class on the projected distance to the nearest neighbor with the neighbor luminosity brighter than $M^{*}=-18$ suggests that spiral arm structures, which are distinguished by arm class, are most affected by local perturbations caused by the nearest massive neighbor galaxy. If the perturbation is strong enough to drive the global density wave in the disk, grand design spiral arms are formed. However, if the perturbations are too strong, they break the symmetry of spiral arms and produce flocculent arms such as a single massive spiral arm.

\section{ACKNOWLEDGMENTS}

This work was supported for two years by Pusan National University Research Grant.

\section{REFERENCES}

Ann, H. B., Park, C., \& Choi, Y.-Y. 2008, Galactic Satellite Systems: Radial Distribution and Environment Dependence of Galaxy Morphology, MNRAS, 389,86

Ann, H. B., \& Lee, H. R. 2013, Spiral Arm Morphology of Nearby Galaxies, JKAS, 46, 141

Bertin, G., Lin, C. C., Lowe, S. A., \& Thurstans, R. P. 1989, Modal Approach to the Morphology of Spiral Galaxies. I - Basic Structure and Astrophysical Viability, ApJ, 338, 738

Bertin, G., Lin, C. C., Lowe, S. A., \& Thurstans, R. P. 1989, Modal Approach to the Morphology of Spiral Galaxies. I - Basic Structure and Astrophysical Viability, ApJ, 338, 1048

Biviano, A., Girardi, M., Giuricin, G., Mardirossian, F., \& Mezzetti, M. 1991, Arm Classification and Velocity Gradients in Spiral Galaxies, ApJ, 376, 458

Choi, I. Y.-G., \& Ann, H. B. 2011, Spiral Arm Morphology in Cluster Environment, JKAS, 44, 161

Choi, Y.-Y., Han, D.-H., \& Kim, S. S. 2010, Korea Institute for Advanced Study Value-Added Galaxy Catalog, JKAS, 43, 191

de Vaucouleurs, G. 1953, On the Distribution of Mass and Luminosity in Elliptical Galaxies, MNRAS, 113, 134

Dressler, A. 1980, Galaxy Morphology in Rich Clusters - Implications for the Formation and Evolution of Galaxies, ApJ, 236, 351

Elmegreen, B. G. 1990, Grand Design, Multiple Arm, and Floccullent Spiral Galaxies, NYASA, 596, 40

Elmegreen, D. M., \& Elmegreen, B. G. 1982, Floccullent and Grand Design Spiral Structure in Field, Binary and Group Galaxies, MNRAS, 201, 1021
Elmegreen, B. G., \& Elmegreen, D. M. 1986, Do Density Waves Trigger Star Formation?, ApJ, 311, 554

Elmegreen, D. M., \& Elmegreen, B. G. 1987, Arm Classifications for Spiral Galaxies, ApJ, 314, 3

Elmegreen, B. G., \& Elmegreen, D. M. 1989, On the Relative Frequency of Flocculent and Grand Gesign Spiral Structures in Barred Galaxies, ApJ, 342, 677

Elmegreen, D. M., Elmegreen, B. G., \& Dressler, A. 1982, Floccullent and Grand Design Spiral Arm Structure in Cluster Galaxies, MNRAS, 201, 1035

Elmegreen, D. M., et al. 2011, Grand Design and Flocculent Spirals in the Spitzer Survey of Stellar Structure in Galaxies (S4G), ApJ, 737, 32

Freeman, K. C. 1970, On the Disks of Spiral and so Galaxies, ApJ, 160, 811

Friedli, D., \& Benz, W. 1993, Secular Evolution of Isolated Barred Galaxies. I - Gravitational Coupling between Stellar Bars and Interstellar Medium, A\&A, 268,65

Friedli, D., \& Benz, W. 1995, Secular Evolution of Isolated Barred Galaxies. II. Coupling between Stars and Interstellar Medium via Star Formation, A\&A, 301,649

Gerola, H., \& Seiden, P. E. 1978, Stochastic Star Formation and Spiral Structure of Galaxies, ApJ, 223, 129

Giuricin, G., Mardirossian, F., \& Mezzetti, M. 1989, Radio Continuum Emission and Arm Classification in Spiral Galaxies, A\&A, 208, 27

Giuricin, G., Monaco, P., Mardirossian, F., \& Mezzetti, M. 1994, The Local Galaxy Density and the Arm Class of Spiral Galaxies, ApJ, 425, 450

Goto, T., Yamauchi, C., Fujita, Y., Okamura, S., Sekiguchi, M., Smail, I., Bernardi, M., \& Gomez, P. L. 2003, The Morphology-Density Relation in the Sloan Digital Sky Survey, MNRAS, 346, 601

Karachentsev, I. D., \& Makarov, D. I. 1999, Galaxy Interactions in the Local Volume, IAUS, 186, 109

Kormendy, J. 1979, A Morphological Survey of Bar, Lens, and Ring Components in Galaxies Secular Evolution in Galaxy Structure, ApJ, 227, 714

Kormendy, J., \& Kennicutt, R. C. Jr. 2004, Secular Evolution and the Formation of Pseudobulges in Disk Galaxies, ARA\&A, 42, 603

Lee, G.-H., Park, C., Lee, M. G., \& Choi, Y.-Y. 2012, Dependence of Barred Galaxy Fraction on Galaxy Properties and Environment, ApJ, 745, 125

Lin, C. C., \& Shu, F. H. 1964, On the Spiral Structure of Disk Galaxies, ApJ, 140, 646

McCall, M. L., \& Schmidt, F. H. 1986, Supernovae in Flocculent and Grand Design Spirals, ApJ, 311, 548

Muldrew, S. I., et al. 2012, Measures of Galaxy Environment - I. What is 'Environment'?, MNRAS, 419, 2670 
Park, C., \& Choi, Y.-Y. 2005, Morphology Segregation of Galaxies in Color-Color Gradient Space, ApJ, 635, L29

Park, C., \& Choi, Y.-Y. 2009, Combined Effects of Galaxy Interactions and Large-Scale Environment on Galaxy Properties, ApJ, 691, 1828

Park, C., Choi, Y.-Y., Vogeley, M. S., Gott, J. R. III., \& Blanton, M. R. 2007, Environmental Dependence of Properties of Galaxies in the Sloan Digital Sky Survey, ApJ, 658, 898

Park, C., Gott, J, R. III., \& Choi, Y.-Y. 2008, Transformation of Morphology and Luminosity Classes of the SDSS Galaxies, ApJ, 6784, 784

Pfenniger, D., \& Norman, C. 1990, Dissipation in Barred Galaxies - The Growth of Bulges and Central Mass Concentrations, ApJ, 363, 391

Romanishin, W. 1985, Star Formation in Grand Design and Flocculent Spiral Galaxies, ApJ, 289, 570

Seiden, P. E., \& Gerola, H. 1982, Propagating Star Formation and the Structure and Evolution of Galaxies, Fund. Cosmic Phys., 7, 241

Stark, A. A., Elmegreen, B. G., \& Chance, D. 1987, Molecules in Galaxies. V - CO Observations of Flocculent and Grand-Design Spirals, ApJ, 322, 64

Tully, R. B., \& Fisher, R. 1977, A New Method of Determining Distances to Galaxies, A\&A, 54, 661

Weinmann, S. M., van den Bosch, F. C., Yang, X., \& Mo, H. J. 2006, Properties of Galaxy Groups in the Sloan Digital Sky Survey - I. The Dependence of Colour, Star Formation and Morphology on Halo Mass, MNRAS, 366, 2

Zhang, X. 1996, Secular Evolution of Spiral Galaxies. I. A Collective Dissipation Process, ApJ, 457, 125

Zhang, X. 1998, Secular Evolution of Spiral Galaxies. II. Formation of Quasi-stationary Spiral Modes, ApJ, 499, 93

Zhang, X. 1999, Secular Evolution of Spiral Galaxies. III. The Hubble Sequence as a Temporal Evolution Sequence, ApJ, 518, 613 